\title{
Avoidance of excisional biopsies in squamous cell carcinoma
}

\author{
S Dobbs, B Anwar, J Goswamy, R Kumar. \\ Manchester University Foundation Trust, Wythenshawe Hospital, Manchester
}

\section{Background}

The most common presenting feature of head and neck squamous cell carcinoma (SCC) is a neck mass; this is also often the first presenting feature of lymphoma. As such; patients may receive the same diagnostic work-up.

The adverse effects of open lymph node biopsy in locally advanced head and neck SCC are historically documented.

Studies analysing prognostic indicators of metastatic SCC in cervical nodes have demonstrated the negative impact of open biopsy on patient survival.'

Patients who undergo initial open biopsy have significantly higher rates of wound necrosis and increased risk of local cervical recurrence and distant metastasis than patients with no biopsy, or biopsy at the time of definitive treatment for head and neck SCC. 2 ,

We reviewed the diagnostic practice of a tertiary referral centre, with the aim to streamline the diagnostic process and avoid ill-considered excision biopsies in patients presenting with a neck mass subsequently diagnosed as SCC.

\section{Methods}

All patients referred to our centre with suspected head and neck lymphoma within the 5-year study period were identified. Retrospective analysis was performed of the pathology records. Date, site, type of histology, and histopathology reports were analysed. Histologically proven cases of SCC diagnosed with open-biopsy were analysed in detail and radiological images studied.

\section{Results}

306 pathology records were reviewed. 2 patients referred as suspected lymphoma underwent open-biopsy which subsequently revealed SCC. Both patients had previous insufficient FNAC samples prior to excision biopsy, and also had imaging with features highly suggestive of SCC.

\section{Discussion}

Fine Needle Aspiration Cytology (FNAC) has an essential role in the diagnostic assessment of head and neck masses in the outpatient clinic setting. FNAC allows the triage of patients into those whom significant disease can be excluded, and those requiring further investigation for malignancy. FNAC can also aid in distinguishing metastatic SCC and cases of possible lymphoma which require lymph node biopsy. However, FNAC can often provide samples that are insufficient for histological diagnosis, requiring further repeat samples to be obtained with subsequent delay in diagnosis, or inappropriate choice to proceed to excision biopsy to obtain results.

NICE guidelines recommend an on-site cytopathologist or biomedical scientist in head and neck clinics as gold standard. By evaluating FNAC sample adequacy on-site, this would allow repeat samples to be taken in the same clinical session, preventing diagnostic delay, and facilitating informed decision making to avoid excision biopsy in possible SCC cases. However this is a significant demand on resources, and is not currently available at our centre. ${ }^{3,4}$

With limitations in the current tools available to provide histological diagnosis, it is important to optimise non-invasive imaging techniques to allow for further evaluation of clinically suspicious neck masses and to assist in distinguishing between SCC and lymphoma. For example, using Computed Tomography Imaging: lymph node necrosis, the absence of distant nodal or extranodal disease and skull base erosion whilst not pathognomic, are suggestive of SCC rather than lymphoma. ${ }^{5}$ Diffusion-weighted Magnetic Resonance Imaging can also aid in distinguishing between SCC and lymphoma, with significantly lower Apparent Diffusion Coefficient values seen in lymphoma vs SCC. 6

\section{Conclusion}

The potential impact of an on-site cytopathologist to evaluate FNAC adequacy in clinic should be further considered in order to provide a gold standard diagnostic pathway. Increased awareness of the radiological features of Head and Neck SCC vs lymphoma will also optimise the diagnostic work up.

I. Jones AS, Cook JA, Phillips DE, Roland NR. Squamous carcinoma presenting as an enlarged cervical lymph node. Cancer. 1993;72:1756-176।

2. McGuirt WF, McCabe BF. Significance of node biopsy before definitive treatment of cervical metastatic carcinoma. Laryngoscope. 1978;88:594-597

3. National Institute for Clinical Excellence. Improving Outcomes in Head and Neck Cancers. London: NICE; 2004.

4. Reddy V, Bennett W, Bassett E, et al. On-site cytotechnician evaluation of the adequacy of fine needle aspiration in a neck lump clinic. Annals of The Royal College of Surgeons of England. 2013;95(8):595-598.

5. Santandreau G, Granell, Gomez-Anson B et al. Imaging of lymphomas of the head and neck. Educational Exhibit, European Society of Radiology, 2013.

6. Maeda M, Kato H, Sakuma $\mathrm{H}$ et al. Usefulness of the apparent diffusion coefficient in line scan diffusion-weighted imaging for distinguishing between squamous cell carcinomas and malignant lymphomas of the head and neck. AJNR Am J Neuroradiol. 2005 May;26(5): I I 86-92 